Suess, E., von Huene, R., et al., 1990

Proceedings of the Ocean Drilling Program, Scientific Results, Vol. 112

\title{
48. GEOCHEMICAL DATA REPORT FOR PERU MARGIN SEDIMENTS FROM SITES 680, 682, 685, AND 6881
}

\author{
Kay-Christian Emeis, ${ }^{2}$ Paul N. Boothe ${ }^{3}$ Barry J. Katz, ${ }^{4}$ John W. Morse,${ }^{3}$ Bob J. Presley, ${ }^{3}$ \\ and Marta T. von Breymann ${ }^{2}$
}

\section{INTRODUCTION}

This data report tabulates results of chemical analyses of sediments from four sites $(680,682,685$, and 688$)$ drilled during Leg 112 offshore Peru. These sediments were recovered from the forearc basins underlying the Peru upwelling area. They are equivalent in facies and age to the Pisco and Monterey formations, both of which are of considerable economic and geological interest as hydrocarbon source rocks deposited under conditions of coastal upwelling. Sediments recovered from the shelf (Site 680) and slope (Sites 682, 685, and 688) during Leg 112 are unconsolidated and are thermally immature. A lack of consolidation and thermal catagenesis makes these deposits ideal targets for chemical investigation into effects of early diagenesis in organic-carbon-rich siliceous muds.

\section{METHODS}

Samples were obtained on board JOIDES Resolution, freeze-dried, ground, and stored in snap-cap glass bottles. Subsamples were used for analysis of calcium carbonate, opal, organic matter abundance and pyrolitic character, iron, and selected trace metal and rare earth elements (REE).

\section{Carbonate}

Carbon dioxide $\left(\mathrm{CO}_{2}\right)$ liberated by acid treatment $(2 \mathrm{~N} \mathrm{HCl})$ of the sample was determined in a Coulometrics $\mathrm{CO}_{2}$ Analyzer and converted to calcium carbonate $\left(\mathrm{CaCO}_{3}\right)$ weight percentages.

\section{Instrumental Neutron Activation Analysis}

Instrumental neutron activation analysis (INAA) was performed in a TRIGA (Training Research Isotope-Production General Atomic) reactor at the Texas A\&M University Nuclear Science Center at a flux of $10^{13}$ neutrons/s. The counting was performed using a germanium-lithium gamma-detector, coupled with a pulse-height multichannel analyzer. These data were reduced and standardized by computer.

\section{Total Reduced Sulfur}

The method employed for determining total reduced sulfur (TRS) is adapted from Zhabina and Volkov (1978) and Canfield et al. (1986). In brief, hydrogen sulfide $\left(\mathrm{H}_{2} \mathrm{~S}\right)$ from reductive decomposition of sulfide by a chromium(II) solution in concentrated hydrochloric acid is titrated with lead perchlo-

\footnotetext{
${ }^{1}$ Suess, E., von Huene, R., et al., 1990. Proc. ODP, Sci. Results, 112: College Station, TX (Ocean Drilling Program).

2 Ocean Drilling Program, Texas A\&M University, College Station, TX 77840 .

${ }^{3}$ Department of Oceanography, Texas A\&M University, College Station, TX 77843 .

${ }^{4}$ Texaco, 3901 Briarpark, Houston, TX 77042.
}

rate and the end point determined with a silver sulfide reference electrode.

\section{Opal}

Opaline silica was determined using a wet alkaline leaching procedure after Eggimann et al. (1980), as modified by Mortlock and Froehlich (in press). After removing organic matter and carbonates that would interfere with the colorimetric determination of silicon, the sample was leached with $2 \mathrm{~N}$ $\mathrm{Na}_{2} \mathrm{CO}_{3}$ and analyzed spectrophotometrically.

\section{Organic Matter}

Ground sediments were used to determine total organic carbon (TOC) by combustion at $600^{\circ} \mathrm{C}$ in the TOC module of a Delsi Nermag Rock Eval II after establishing the yields of hydrocarbons $\left(\mathrm{S}_{1}\right.$ and $\left.\mathrm{S}_{2}\right)$ and $\mathrm{CO}_{2}\left(\mathrm{~S}_{3}\right)$ per gram of sediment in a programmed pyrolysis run. The procedure is described in detail in Espitalié et al. (1985), who also gave guidelines for interpreting results. Some considerations as to the usefulness of pyrolysis in immature sediments and some pitfalls are outlined in Katz (1983) and Peters (1986). Samples of Holes $682 \mathrm{~A}$ and $685 \mathrm{~A}$ were not analyzed by pyrolysis. Here, we employed a Perkin Elmer Model 240C CHN analyzer to determine elemental carbon and nitrogen by combustion at $1000^{\circ} \mathrm{C}$. Organic carbon was determined by subtracting carbonate carbon from total carbon.

\section{RESULTS}

Results of the chemical analyses are listed for individual sites in Tables 1 through 4 . A composite data set of all analyses yielded average values that are compared with available literature data on sediments of other upwellingrelated facies, of organic carbon-rich rocks from different depositional settings, and with "average shale" and "average carbonates" in Table 5. Analysis and interpretation of these results are included in Emeis and Morse (this volume) and von Breymann et al. (this volume).

\section{REFERENCES}

Brumsack, H. J., and Gieskes, J. M., 1983. Interstitial water trace element chemistry of laminated sediments from the Gulf of California, Mexico. Mar. Chem., 14:89-106.

Canfield, D. E., Raiswell, R., Westrich, J. T., Reaves, C. M., and Berner, R. A., 1986. The use of chromium reduction in the analysis of reduced inorganic sulfur in sediments and shales. Chem. Geol., 54:149-155.

Eggimann, D. W., Manheim, F. T., and Betzer, P. R., 1980. Dissolution and analysis of amorphous silica in marine sediments. $J$. Sediment. Petrol., 50:215-225.

Emeis, K.-C., 1985. Geochemie und Fazies von Schwarzschiefern und organischreichen Sedimenten des Südatlantik [ $\mathrm{PhD}$ dissert.]. Hamburg Univ., Hamburg, F.R.G. 
Espitalié, J., Deroo, G., and Marquis, F., 1985. La pyrolyse RockEval et ses applications. Rev. Inst. Français Pet., 40:563-579.

Katz, B. J., 1983. Limitations of 'Rock-Eval' pyrolysis for typing organic matter. Org. Geochem., 4:195-199.

Peters, K. E., 1986. Guidelines for evaluating petroleum source rocks using programmed pyrolysis. AAPG Bull., 70:318-329.

Mortlock, R. A., and Froehlich, P. N., in press. A simple method for the rapid determination of biogenic opal in marine sediments. $J$. Sediment. Petrol.

Turekian, K. K., and Wedepohl, K. H., 1961. Distribution of the elements in some major units of the earth's crust. Geol. Soc. Am. Bull., 72:175-192.
Zhabina, N. N., and Volkov, I. I., 1978. A method of determination of various sulfur compounds in sea sediments and rocks. In Krumbein, W. E. (Ed.), Environmental Biogeochemistry and Geomicrobiology, Vol. 3: Methods, Metals, and Assessment: Ann Arbor (Ann Arbor Scientific Publ.), 735-746.

Date of initial receipt: 24 April 1989

Date of acceptance: 7 June 1989

Ms 112B-203

Table 1. Sediment chemistry at Hole 680 .

\begin{tabular}{|c|c|c|c|c|c|c|c|c|c|c|c|c|}
\hline $\begin{array}{l}\text { Core, section, } \\
\text { interval }(\mathrm{cm})\end{array}$ & $\begin{array}{l}\text { Depth } \\
\text { (mbsf) }\end{array}$ & Opal-Si & $\begin{array}{c}\mathrm{CaCO}_{3} \\
(\%)\end{array}$ & $\begin{array}{l}\mathrm{T}_{\max } \\
\left({ }^{\circ} \mathrm{C}\right)\end{array}$ & $\begin{array}{c}\mathrm{S}_{1} \\
(\mathrm{mg} / \mathrm{g})\end{array}$ & $\begin{array}{c}\mathrm{S}_{2} \\
(\mathrm{mg} / \mathrm{g})\end{array}$ & $\begin{array}{c}\mathrm{S}_{3} \\
(\mathrm{mg} / \mathrm{g})\end{array}$ & $\begin{array}{l}\text { TOC } \\
(\%)\end{array}$ & $\begin{array}{l}\text { TRS } \\
(\%)\end{array}$ & $\underset{(\mathrm{ppm})}{\mathrm{La}}$ & $\underset{(\mathrm{ppm})}{\mathrm{Ce}}$ & $\begin{array}{c}\mathrm{Sm} \\
(\mathrm{ppm})\end{array}$ \\
\hline $680 \mathrm{~A}-01 \mathrm{H}-2,88-90$ & 2.40 & 4.2 & 0.75 & 399 & 25.30 & 92.20 & 34.10 & 12.42 & 0.77 & 21 & 36 & 5.1 \\
\hline 01H-2, 104-106 & 2.60 & n.d. & 0.83 & 405 & 5.10 & 28.40 & 5.40 & 5.31 & 1.03 & 22 & 38 & 5.0 \\
\hline $01 \mathrm{H}-4,7779$ & 5.30 & 6.8 & 0.50 & 394 & 6.10 & 25.60 & 6.10 & 6.89 & 0.63 & 22 & 37 & 4.8 \\
\hline $01 \mathrm{H}-6,37-39$ & 7.90 & 5.9 & 1.83 & n.d. & n.d. & n.d. & n.d. & n.d. & 0.78 & 13 & 23 & 3.8 \\
\hline $02 \mathrm{H}-1,86-88$ & 9.20 & 13.6 & 12.00 & 406 & 4.80 & 26.40 & 6.40 & 5.01 & 0.84 & 21 & 34 & 4.6 \\
\hline $02 \mathrm{H}-2,96-98$ & 10.80 & 5.4 & 31.99 & 401 & 1.90 & 12.40 & 3.00 & 3.38 & 1.14 & 39 & 69 & 8.0 \\
\hline $02 \mathrm{H}-3,51-53$ & 11.80 & 8.2 & 0.58 & 390 & 6.60 & 34.50 & 6.00 & 6.86 & 0.94 & 26 & 43 & 6.3 \\
\hline $02 \mathrm{H}-4,25-26$ & 13.10 & 2.8 & 0.00 & 385 & 4.70 & 30.40 & 5.10 & 7.12 & 1.07 & 24 & 40 & 5.3 \\
\hline $02 \mathrm{H}-5,25-27$ & 14.60 & 3.9 & 11.83 & 397 & 2.00 & 13.90 & 3.60 & 1.95 & 0.92 & 22 & 43 & 5.3 \\
\hline $02 \mathrm{H}-6,25-26$ & 16.10 & 5.4 & 0.08 & 402 & 3.50 & 24.70 & 3.80 & 3.47 & 0.96 & 23 & 40 & 5.3 \\
\hline $02 \mathrm{H}-6,68-70$ & 16.40 & n.d. & 0.33 & 406 & 2.60 & 23.90 & 3.50 & 3.16 & 1.21 & 27 & 46 & 5.8 \\
\hline $02 \mathrm{H}-7,44-46$ & 17.70 & 7.9 & 15.25 & 390 & 8.50 & 42.60 & 7.00 & 4.54 & 1.00 & 26 & 41 & 6.6 \\
\hline $03 \mathrm{H}-1,93-95$ & 18.80 & n.d. & 14.74 & n.d. & n.d. & n.d. & n.d. & n.d. & 0.94 & 25 & 47 & 5.9 \\
\hline $03 \mathrm{H}-2,25-27$ & 19.60 & 3.4 & 3.76 & 412 & 4.30 & 28.60 & 5.60 & 3.77 & 0.75 & 14 & 25 & 3.4 \\
\hline $03 \mathrm{H}-3,116-118$ & 22.00 & 6.4 & 10.75 & 400 & 8.50 & 42.10 & 8.70 & 7.26 & 0.96 & 20 & 33 & 5.0 \\
\hline $03 \mathrm{H}-4,25-27$ & 22.60 & 7.3 & 1.00 & 413 & 5.40 & 40.40 & 7.40 & 5.80 & 0.84 & 11 & 19 & 2.5 \\
\hline $03 \mathrm{H}-5,32-34$ & 24.10 & 2.9 & 3.17 & 404 & 1.20 & 8.80 & 2.80 & 4.07 & 1.01 & 28 & 52 & 6.1 \\
\hline $03 \mathrm{H}-6,25-27$ & 25.60 & n.d. & 26.49 & 425 & 0.20 & 2.30 & 1.30 & 2.00 & 0.18 & 31 & 55 & 6.8 \\
\hline $03 \mathrm{H}-7,25-27$ & 27.10 & n.d. & 18.16 & 403 & 4.20 & 28.50 & 6.40 & 4.17 & 0.77 & 21 & 33 & 5.2 \\
\hline $04 \mathrm{H}-1,25-26$ & 27.60 & 6.0 & 29.82 & 411 & 2.00 & 15.60 & 4.10 & 3.89 & 0.41 & 19 & 30 & 4.3 \\
\hline $04 \mathrm{H}-2,25-26$ & 29.10 & n.d. & 27.66 & 407 & 5.00 & 42.30 & 4.50 & 5.70 & 0.63 & 22 & 34 & 7.1 \\
\hline $04 \mathrm{H}-3,25-26$ & 30.60 & n.d. & 0.17 & 401 & 6.00 & 43.10 & 4.50 & 10.09 & 1.07 & 25 & 43 & 5.7 \\
\hline $04 \mathrm{H}-4,25-26$ & 32.10 & 6.5 & 0.67 & 399 & 5.50 & 35.60 & 4.80 & 7.35 & 0.92 & 21 & 36 & 5.6 \\
\hline $04 \mathrm{H}-5,25-26$ & 33.60 & 2.7 & 7.25 & 421 & 0.60 & 7.50 & 2.20 & 2.24 & 0.41 & 26 & 45 & 5.7 \\
\hline $04 \mathrm{H}-6,25-26$ & 35.10 & n.d. & 20.66 & 413 & 1.80 & 15.40 & 3.30 & 2.88 & 0.62 & 26 & 45 & 5.9 \\
\hline $04 \mathrm{H}-7,25-25$ & 36.60 & 1.9 & 0.00 & 405 & 2.40 & 21.40 & 2.90 & 3.71 & 0.94 & 28 & 51 & 6.3 \\
\hline $05 \mathrm{H}-1,18-19$ & 37.00 & 4.2 & 4.83 & 398 & 3.10 & 22.70 & 3.10 & 4.72 & 0.91 & 25 & 44 & 6.0 \\
\hline $05 \mathrm{H}-2,18-19$ & 38.50 & 10.0 & 2.50 & 387 & 6.20 & 33.60 & 4.50 & 8.23 & 1.05 & 31 & 45 & 5.9 \\
\hline $05 \mathrm{H}-3,57-59$ & 40.40 & 2.4 & 7.33 & n.d. & n.d. & n.d. & n.d. & n.d. & 1.29 & 31 & 59 & 6.6 \\
\hline $05 \mathrm{H}-4,17-18$ & 41.50 & 7.3 & 0.17 & 388 & 4.70 & 27.40 & 4.30 & 6.24 & 0.99 & 19 & 32 & 4.2 \\
\hline $05 \mathrm{H}-5,17-18$ & 43.00 & n.d. & 2.17 & 396 & 3.00 & 26.40 & 4.40 & 5.44 & 0.98 & 38 & 61 & 7.9 \\
\hline $06 \mathrm{H}-1,18-19$ & 46.40 & 7.3 & 3.84 & 385 & 3.80 & 20.10 & 2.60 & 4.40 & 1.23 & 22 & 43 & 4.9 \\
\hline $07 \mathrm{H}-1,35-36$ & 56.20 & 12.3 & 0.42 & 387 & 3.30 & 16.70 & 2.40 & 3.68 & 0.67 & 16 & 27 & 4.2 \\
\hline $07 \mathrm{H}-2,80-82$ & 58.10 & 2.1 & 1.75 & n.d. & n.d. & n.d. & n.d. & n.d. & 1.60 & 30 & 54 & 5.8 \\
\hline $07 \mathrm{H}-3,34-36$ & 59.10 & 1.2 & 6.75 & 329 & 0.00 & 0.10 & 0.70 & 0.18 & 1.81 & 31 & 57 & 6.0 \\
\hline $07 \mathrm{H}-4,33-34$ & 60.60 & 1.8 & 4.42 & 385 & 0.10 & 0.30 & 1.00 & 0.03 & 1.94 & 29 & 55 & 5.9 \\
\hline $07 \mathrm{H}-5,34-36$ & 62.10 & 1.4 & 3.67 & 335 & 0.10 & 0.40 & 0.90 & 2.74 & 1.94 & 30 & 55 & 5.9 \\
\hline $07 \mathrm{H}-6,27-29$ & 63.60 & 3.3 & 2.00 & 384 & 0.10 & 0.70 & 0.60 & 2.16 & 1.93 & 26 & 51 & 5.6 \\
\hline $08 \mathrm{H}-1,34-36$ & 65.70 & 1.5 & 2.45 & 389 & 0.20 & 1.10 & 1.30 & 2.71 & 1.83 & 28 & 52 & 5.5 \\
\hline $08 \mathrm{H}-2,35-37$ & 67.20 & 3.6 & 4.83 & 377 & 0.60 & 1.90 & 0.90 & 4.20 & 1.56 & 23 & 46 & 4.7 \\
\hline $08 \mathrm{H}-3,34-36$ & 68.70 & 3.4 & 1.36 & 387 & 1.20 & 4.80 & 1.30 & 5.02 & 1.81 & 27 & 49 & 5.5 \\
\hline $08 \mathrm{H}-4,34-36$ & 70.10 & 3.6 & 1.25 & 381 & 1.30 & 5.00 & 1.40 & 5.04 & 1.66 & 22 & 40 & 4.5 \\
\hline $08 \mathrm{H}-5,34-36$ & 71.70 & 6.8 & 0.42 & 383 & 1.80 & 6.40 & 1.40 & 6.13 & n.d. & 18 & 34 & 3.8 \\
\hline $08 \mathrm{H}-6,34-36$ & 73.20 & 1.1 & 5.50 & n.d. & n.d. & n.d. & n.d. & n.d. & n.d. & 24 & 45 & 4.8 \\
\hline $08 \mathrm{H}-7,34-36$ & 74.70 & 3.7 & 1.07 & n.d. & n.d. & n.d. & n.d. & 6.01 & n.d. & 23 & 42 & 4.9 \\
\hline $09 \mathrm{H}-1,34-36$ & 75.10 & 1.3 & 1.33 & n.d. & n.d. & n.d. & n.d. & 3.13 & n.d. & 32 & 58 & 6.6 \\
\hline $09 \mathrm{H}-2,34-36$ & 76.60 & 3.1 & 0.25 & n.d. & n.d. & n.d. & n.d. & 3.65 & n.d. & 27 & 53 & 5.8 \\
\hline $09 \mathrm{H}-3,34-36$ & 78.10 & 3.1 & 0.83 & n.d. & n.d. & n.d. & n.d. & 6.97 & n.d. & 21 & 37 & 4.4 \\
\hline
\end{tabular}


Table 1 (continued).

\begin{tabular}{|c|c|c|c|c|c|c|c|c|c|c|c|c|c|}
\hline$\underset{(\mathrm{ppm})}{\mathrm{Eu}}$ & $\begin{array}{c}\mathrm{Yb} \\
(\mathrm{ppm})\end{array}$ & $\underset{(\mathrm{ppm})}{\mathrm{Lu}}$ & $\underset{(\mathrm{ppm})}{\mathrm{Ba}}$ & $\underset{(\mathrm{ppm})}{\mathrm{Cr}}$ & $\begin{array}{l}\mathrm{Fe} \\
(\%)\end{array}$ & $\underset{(\mathrm{ppm})}{\mathrm{Cs}}$ & $\underset{(\mathrm{ppm})}{\mathrm{Co}}$ & $\underset{(\mathrm{ppm})}{\mathrm{Hf}}$ & $\begin{array}{c}\mathrm{Rb} \\
(\mathrm{ppm})\end{array}$ & $\underset{(\mathrm{ppm})}{\mathrm{Sc}}$ & $\begin{array}{c}\text { Th } \\
\text { (ppm) }\end{array}$ & $\begin{array}{c}\text { Sb } \\
(\mathrm{ppm})\end{array}$ & $\underset{(\mathrm{ppm})}{\mathrm{Br}}$ \\
\hline 0.73 & 0.1 & 0.50 & 401 & 116 & 1.93 & 5.1 & 6.0 & 4.4 & 53 & 8.4 & 6.6 & 2.8 & 331 \\
\hline 0.76 & 2.0 & 0.49 & 396 & 118 & 2.12 & 6.8 & 6.4 & 4.5 & 61 & 8.8 & 6.8 & 2.7 & 307 \\
\hline 0.72 & 2.0 & 0.46 & 332 & 90 & 2.17 & 7.2 & 5.9 & 4.1 & 61 & 8.9 & 6.8 & 2.4 & 196 \\
\hline 0.48 & 1.3 & 0.42 & 374 & 127 & 1.59 & 4.2 & 5.5 & 2.1 & 40 & 6.8 & 4.2 & 1.7 & 375 \\
\hline 0.70 & 1.9 & 0.40 & 463 & 116 & 1.89 & 6.7 & 6.5 & 3.1 & 58 & 8.3 & 6.0 & 1.4 & 275 \\
\hline 1.31 & 3.3 & 0.71 & 568 & 130 & 3.96 & 14.7 & 11.8 & 7.6 & 121 & 16.3 & 13.1 & 4.0 & 208 \\
\hline 0.84 & 1.7 & 0.55 & 374 & 139 & 2.30 & 10.4 & 6.7 & 3.1 & 79 & 10.9 & 7.0 & 2.1 & 215 \\
\hline 0.76 & 2.1 & 0.51 & 362 & 127 & 2.52 & 9.4 & 7.2 & 3.3 & 72 & 11.2 & 7.3 & 2.4 & 231 \\
\hline 0.95 & 2.5 & 0.46 & 427 & 93 & 2.50 & 6.6 & 6.2 & 5.2 & 67 & 10.4 & 7.7 & 2.4 & 140 \\
\hline 0.70 & 1.8 & 0.51 & 337 & 124 & 2.07 & 9.4 & 6.6 & 3.8 & 67 & 9.0 & 6.7 & 2.3 & 247 \\
\hline 0.87 & 2.4 & 0.49 & 344 & 104 & 2.41 & 11.4 & 7.3 & 4.2 & 84 & 11.1 & 8.1 & 1.4 & 171 \\
\hline 0.78 & 2.3 & 0.55 & 457 & 163 & 2.35 & 9.9 & 7.5 & 3.5 & 80 & 10.2 & 7.2 & 2.6 & 318 \\
\hline 0.92 & 2.6 & 0.53 & 375 & 126 & 2.29 & 8.4 & 6.1 & 4.7 & 72 & 9.9 & 7.8 & 2.2 & 248 \\
\hline 0.44 & 1.3 & 0.37 & 204 & 96 & 1.42 & 5.2 & 4.2 & 1.9 & 43 & 6.2 & 4.4 & 1.9 & 258 \\
\hline 0.64 & 1.9 & 0.45 & 388 & 154 & 2.06 & 5.9 & 6.3 & 3.1 & 59 & 8.9 & 6.0 & 2.6 & 251 \\
\hline 0.45 & 1.1 & 0.27 & 182 & 105 & 1.36 & 4.1 & 4.6 & 1.7 & 38 & 5.3 & 3.6 & 1.8 & 280 \\
\hline 1.05 & 2.6 & 0.53 & 431 & 85 & 2.57 & 7.3 & 9.9 & 1.1 & 95 & 11.0 & 8.9 & 2.5 & 82 \\
\hline 1.22 & 3.4 & 0.67 & 574 & 58 & 1.82 & 4.7 & 5.1 & 10.0 & 76 & 9.5 & 8.4 & 2.2 & 52 \\
\hline 0.67 & 1.9 & 0.48 & 228 & 122 & 1.93 & 6.8 & 5.5 & 3.2 & 58 & 8.1 & 6.1 & 2.1 & 187 \\
\hline 0.66 & 2.2 & 0.39 & 295 & 83 & 1.19 & 3.9 & 3.5 & 3.9 & 44 & 5.8 & 5.3 & 1.9 & 166 \\
\hline 0.66 & 2.0 & 0.99 & 413 & 200 & 1.71 & 5.9 & 6.0 & 2.2 & 47 & 8.1 & 5.2 & 2.2 & 540 \\
\hline 0.83 & 2.3 & 0.53 & 396 & 174 & 2.56 & 11.7 & 8.5 & 4.3 & 78 & 11.3 & 8.4 & 2.2 & 352 \\
\hline 0.71 & 1.9 & 0.52 & 347 & 130 & 1.93 & 7.3 & 6.3 & 3.0 & 60 & 8.5 & 6.3 & 1.7 & 267 \\
\hline 0.96 & 3.0 & 0.56 & 429 & 64 & 1.27 & 3.1 & 4.1 & 10.0 & 45 & 7.1 & 7.0 & 2.0 & 103 \\
\hline 1.02 & 3.0 & 0.62 & 367 & 97 & 1.84 & 5.8 & 5.0 & 6.7 & 68 & 8.8 & 7.6 & 2.5 & 130 \\
\hline 1.02 & 2.8 & 0.57 & 420 & 126 & 2.33 & 9.0 & 7.2 & 6.6 & 74 & 11.1 & 8.9 & 2.0 & 199 \\
\hline 0.88 & 2.6 & 0.55 & 409 & 112 & 2.10 & 9.5 & 6.8 & 5.3 & 64 & 9.6 & 7.5 & 2.2 & 246 \\
\hline 1.02 & 3.0 & 0.62 & 326 & 129 & 2.10 & 8.0 & 7.0 & 3.0 & 59 & 8.0 & 6.0 & 2.0 & 234 \\
\hline 1.13 & 2.8 & 0.51 & 402 & 122 & 2.93 & 13.8 & 9.2 & 4.5 & 87 & 13.9 & 9.8 & 2.8 & 179 \\
\hline 0.63 & 1.5 & 0.37 & 270 & 96 & 1.85 & 10.0 & 8.2 & 2.5 & 61 & 7.1 & 5.4 & 2.0 & 172 \\
\hline 1.26 & 3.4 & 0.72 & 595 & 164 & 3.39 & 10.3 & 12.0 & 6.3 & 88 & 13.4 & 10.6 & 2.4 & 338 \\
\hline 0.87 & 2.3 & 0.43 & 452 & 117 & 2.50 & 12.6 & 7.4 & 4.5 & 76 & 10.4 & 8.0 & 2.3 & 202 \\
\hline 0.55 & 1.4 & 0.40 & 309 & 85 & 1.35 & 5.4 & 4.4 & 2.1 & 38 & 5.3 & 4.3 & 1.1 & 210 \\
\hline 1.23 & 2.9 & 0.47 & 391 & 43 & 3.81 & 12.4 & 11.6 & 5.0 & 109 & 13.9 & 10.2 & 3.7 & 37 \\
\hline 1.12 & 2.6 & 0.48 & 426 & 45 & 4.11 & 9.8 & 11.9 & 5.1 & 110 & 14.2 & 10.3 & 4.5 & 31 \\
\hline 1.15 & 2.6 & 0.48 & 430 & 48 & 3.86 & 11.5 & 11.0 & 4.6 & 102 & 12.8 & 10.1 & 2.2 & 44 \\
\hline 1.17 & 2.5 & 0.45 & 424 & 48 & 4.17 & 12.2 & 12.2 & 4.4 & 122 & 14.3 & 10.4 & 3.6 & 43 \\
\hline 0.97 & 2.4 & 0.41 & 418 & 51 & 3.72 & 11.7 & 9.9 & 4.3 & 89 & 12.9 & 9.9 & 1.9 & 56 \\
\hline 1.08 & 2.3 & 0.44 & 432 & 59 & 3.59 & 10.2 & 9.4 & 4.4 & 108 & 12.7 & 10.1 & 2.7 & 59 \\
\hline 0.88 & 2.1 & 0.36 & 318 & 57 & 2.87 & 10.9 & 8.8 & 3.6 & 85 & 11.0 & 8.5 & 1.3 & 116 \\
\hline 0.96 & 2.2 & 0.44 & 373 & 90 & 3.40 & 10.4 & 9.2 & 3.8 & 104 & 12.2 & 8.4 & 2.7 & 69 \\
\hline 0.84 & 1.9 & 0.33 & 320 & 83 & 3.16 & 10.6 & 9.0 & 3.7 & 76 & 11.9 & 8.3 & 1.4 & 113 \\
\hline 0.71 & 1.6 & 0.30 & 272 & 86 & 2.63 & 7.4 & 7.0 & 2.8 & 72 & 8.5 & 6.4 & 1.6 & 124 \\
\hline 0.89 & 2.0 & 0.37 & 478 & 49 & 2.68 & 7.2 & 7.4 & 4.5 & 74 & 9.9 & 8.6 & 1.5 & 53 \\
\hline 0.85 & 2.0 & 0.38 & 360 & 119 & 3.46 & 9.8 & 8.5 & 3.9 & 97 & 11.0 & 8.7 & 2.1 & 89 \\
\hline 1.21 & 3.0 & 0.55 & 451 & 74 & 4.38 & 11.6 & 11.3 & 4.4 & 120 & 15.4 & 10.6 & 3.0 & 54 \\
\hline 1.05 & 2.9 & 0.48 & 418 & 77 & 3.86 & 12.4 & 10.3 & 4.1 & 98 & 14.5 & 10.0 & 1.8 & 68 \\
\hline 0.78 & 1.8 & 0.33 & 343 & 124 & 3.73 & 9.7 & 9.2 & 3.4 & 95 & 11.8 & 8.5 & 2.3 & 90 \\
\hline
\end{tabular}


Table 2. Sediment chemistry at Site $\mathbf{6 8 2}$.

\begin{tabular}{|c|c|c|c|c|c|c|c|c|c|c|c|c|c|c|c|}
\hline Sample ID & $\begin{array}{l}\text { Depth } \\
\text { (mbsf) }\end{array}$ & $\begin{array}{c}\text { Opal-Si } \\
(\%)\end{array}$ & $\begin{array}{c}\mathrm{CaCO}_{3} \\
(\%)\end{array}$ & $\begin{array}{l}\text { TOC } \\
(\%)\end{array}$ & $\begin{array}{l}\text { TN } \\
(\%)\end{array}$ & $\begin{array}{l}\text { TRS } \\
(\%)\end{array}$ & $\mathrm{C} / \mathrm{N}$ & $\underset{(\mathrm{ppm})}{\mathrm{La}}$ & $\begin{array}{c}\mathrm{Ce} \\
(\mathrm{ppm})\end{array}$ & $\underset{(\mathrm{ppm})}{\mathrm{Sm}}$ & $\begin{array}{c}\text { Eu } \\
(\mathrm{ppm})\end{array}$ & $\begin{array}{c}\mathrm{Yb} \\
(\mathrm{ppm})\end{array}$ & $\begin{array}{c}\mathrm{Lu} \\
(\mathrm{ppm})\end{array}$ & $\begin{array}{c}\mathrm{Ba} \\
(\mathrm{ppm})\end{array}$ & $\begin{array}{c}\mathrm{Cr} \\
\text { (ppm) }\end{array}$ \\
\hline $01 \mathrm{H}-3,76-78$ & 3.76 & 3.1 & 12.75 & 5.94 & 0.35 & 0.54 & 16.97 & 31 & 52 & 6.0 & 0.99 & 2.8 & 0.49 & 1035 & 74 \\
\hline $01 \mathrm{H}-6,56-58$ & 8.06 & 3.2 & 0.92 & 6.86 & 0.62 & 0.58 & 11.06 & 32 & 52 & 6.5 & 1.07 & 3.2 & 0.66 & 2245 & 108 \\
\hline $02 \mathrm{H}-3,45-47$ & 13.25 & 3.4 & 1.50 & 15.71 & 0.78 & 0.84 & 20.14 & 24 & 37 & 5.0 & 0.80 & 2.5 & 0.53 & 1755 & 77 \\
\hline $03 \mathrm{H}-2,55-57$ & 21.35 & 4.2 & 3.83 & 9.69 & 0.53 & 1.03 & 18.28 & 24 & 40 & 4.6 & 0.79 & 2.2 & 0.40 & 1718 & 102 \\
\hline $03 \mathrm{H}-5,67-77$ & 25.97 & 4.7 & 0.83 & 8.25 & n.d. & 1.27 & n.d. & 26 & 46 & 5.2 & 0.90 & 2.4 & 0.47 & 1391 & 125 \\
\hline $04 \mathrm{H}-1,127-128$ & 30.07 & 12.6 & 0.25 & 17.94 & 1.18 & n.d. & n.d. & 15 & 25 & 3.3 & 0.50 & 1.4 & 0.31 & 1198 & 90 \\
\hline $05 \mathrm{H}-1,48-49$ & 38.88 & 12.1 & n.d. & 11.41 & 0.73 & 0.80 & 15.63 & 16 & 26 & 3.3 & 0.49 & 1.3 & 0.29 & 989 & 74 \\
\hline $06 \mathrm{H}-3,140-150$ & 52.30 & 10.9 & 7.01 & 8.10 & n.d. & 1.17 & n.d. & 16 & 27 & 3.4 & 0.57 & 1.7 & 0.44 & 670 & 114 \\
\hline $07 \mathrm{H}-2,79-81$ & 58.09 & 5.6 & 0.00 & 10.35 & 0.77 & 1.46 & 13.44 & 21 & 38 & 3.8 & 0.75 & 1.7 & 0.36 & 3208 & 114 \\
\hline $08 \mathrm{H}-1,46-48$ & 67.26 & 13.2 & 2.75 & 15.22 & 0.98 & 0.81 & 15.53 & 12 & 21 & 2.6 & 0.39 & 1.2 & 0.26 & 432 & 120 \\
\hline $09 \mathrm{H}-1,106-116$ & 77.50 & 15.7 & 2.08 & 7.14 & 1.12 & 1.02 & 6.38 & 11 & 20 & 2.5 & 0.44 & 1.0 & 0.27 & 385 & 138 \\
\hline $10 \mathrm{H}-1,94-96$ & 86.74 & 17.6 & 1.42 & 13.61 & 1.40 & 0.19 & 9.72 & 7 & 11 & 1.9 & 0.24 & n.d. & 0.26 & 307 & 91 \\
\hline $12 X-1,140-150$ & 106.30 & 6.2 & 1.67 & 2.25 & n.d. & 1.80 & n.d. & 18 & 34 & 3.8 & 0.74 & 1.5 & 0.32 & 585 & 130 \\
\hline $14 \mathrm{X}-\mathrm{CC}, 2-3$ & 126.58 & 12.2 & 3.00 & 7.88 & 0.57 & 0.98 & 13.82 & 11 & 19 & 2.5 & 0.50 & 0.9 & 0.22 & 471 & 117 \\
\hline $15 X-1,134-139$ & 134.70 & 11.4 & 4.50 & 3.65 & 0.66 & 0.83 & 5.53 & 11 & 19 & 2.4 & 0.41 & 1.0 & 0.22 & 366 & 146 \\
\hline $17 \mathrm{H}-1,146-147$ & 153.76 & n.d. & 9.50 & 11.05 & 0.61 & 1.06 & 18.11 & 17 & 29 & 3.8 & 0.63 & 1.4 & 0.30 & 533 & 149 \\
\hline $18 X-1,145-150$ & 163.30 & 12.7 & 6.25 & 9.56 & 0.53 & n.d. & 18.04 & 11 & 18 & 2.7 & 0.44 & 1.0 & 0.26 & 376 & 122 \\
\hline $18 X-2,84-86$ & 164.14 & 13.6 & 7.58 & 10.01 & 0.55 & 0.81 & 18.20 & 11 & 20 & 3.0 & 0.42 & 1.0 & 0.53 & 415 & 121 \\
\hline $20 X-1,59-61$ & 181.39 & 12.9 & 5.17 & 8.82 & 0.49 & 0.94 & 18.00 & 11 & 18 & 2.6 & 0.43 & 0.9 & 0.18 & 379 & 106 \\
\hline $20 X-2,44-46$ & 182.74 & 12.9 & 6.08 & 9.66 & 0.74 & 1.22 & 13.05 & 10 & 16 & 2.3 & 0.41 & 0.9 & 0.19 & 385 & 116 \\
\hline $21 X-1,93-95$ & 191.23 & n.d. & 8.25 & 8.88 & 0.57 & 0.90 & 15.58 & 10 & 16 & 2.4 & 0.39 & 0.9 & 0.19 & 429 & 100 \\
\hline $22 X-2,63-64$ & 201.93 & n.d. & 4.67 & 7.88 & 0.54 & 0.86 & 14.59 & 16 & 25 & 3.6 & 0.63 & 1.6 & 0.27 & 538 & 109 \\
\hline $24 X-1,117-120$ & 219.97 & 11.0 & 7.08 & 8.31 & 0.48 & 0.87 & 17.31 & 18 & 25 & 3.7 & 0.62 & 1.5 & 0.31 & 527 & 111 \\
\hline $25 \mathrm{X}-\mathrm{CC}, 18-20$ & 229.17 & n.d. & 7.92 & 8.72 & 0.47 & 0.70 & 18.55 & 15 & 24 & 3.9 & 0.56 & 1.4 & 0.35 & 537 & 107 \\
\hline $26 \mathrm{X}-\mathrm{CC}, 11-13$ & 238.80 & 14.4 & 7.83 & 9.21 & 0.63 & 0.80 & 14.62 & 13 & 18 & 2.8 & 0.39 & 0.9 & 0.24 & 437 & 98 \\
\hline $27 \mathrm{X}-1,106-113$ & 248.40 & 14.8 & 5.39 & 3.19 & n.d. & 0.77 & n.d. & 12 & 18 & 2.6 & 0.39 & 0.8 & 0.24 & 383 & 97 \\
\hline $28 X-1,6-7$ & 256.86 & n.d. & 6.42 & 8.02 & 0.30 & 0.45 & 26.73 & 14 & 19 & 2.9 & 0.44 & 1.0 & 0.25 & 466 & 84 \\
\hline $29 X-1,16-17$ & 266.46 & n.d. & 5.92 & 10.02 & 0.43 & 0.57 & 23.30 & 8 & 11 & 2.1 & 0.29 & 0.5 & 0.13 & 304 & 76 \\
\hline $33 X-1,16-17$ & 295.26 & n.d. & 3.42 & 11.89 & 0.92 & n.d. & 12.92 & 18 & 26 & 4.4 & 0.55 & 1.3 & 0.36 & 537 & 146 \\
\hline $34 X-2,140-150$ & 307.60 & 4.2 & 13.20 & 3.72 & n.d. & 1.82 & n.d. & 23 & 34 & 4.9 & 1.01 & 2.4 & 0.58 & 755 & 202 \\
\hline $34 X-3,30-32$ & 307.90 & n.d. & 7.42 & 6.14 & 0.51 & 2.05 & 12.04 & 19 & 33 & 4.4 & 0.81 & 2.0 & 0.45 & 584 & 186 \\
\hline $35 X-2,97-98$ & 313.67 & n.d. & 2.83 & 4.27 & 0.46 & 2.63 & 9.28 & 19 & 32 & 4.4 & 0.85 & 2.0 & 0.40 & 608 & 138 \\
\hline $36 X-1,80-85$ & 321.46 & n.d. & 3.58 & 5.16 & 0.39 & 1.74 & 13.23 & 20 & 36 & 4.5 & 0.92 & 1.7 & 0.43 & 725 & 120 \\
\hline $37 X-2,83-84$ & 332.52 & n.d. & 7.83 & 6.78 & 0.48 & 1.29 & 14.13 & 19 & 32 & 4.5 & 0.73 & 1.6 & 0.37 & 643 & 134 \\
\hline $39 X-2,131-141$ & 352.20 & n.d. & 7.59 & 2.97 & n.d. & 1.72 & n.d. & 17 & 25 & 5.0 & 0.67 & 1.3 & 0.55 & 666 & 149 \\
\hline $40 \times-2,9-11$ & 360.29 & 4.7 & 5.50 & 6.09 & 0.43 & 1.72 & 14.16 & 19 & 34 & 4.4 & 0.90 & 1.8 & 0.49 & 725 & 136 \\
\hline $41 X-C C, 10-11$ & 368.30 & n.d. & 3.17 & 12.92 & 0.65 & 2.13 & 19.88 & 15 & 24 & 4.4 & 0.66 & 1.7 & 0.49 & 474 & 203 \\
\hline $42 X-C C, 12-13$ & 378.00 & n.d. & 0.75 & 7.37 & $0.4 \mathrm{~B}$ & 0.95 & 15.35 & 9 & 13 & 2.4 & 0.36 & 1.0 & 0.02 & 322 & 121 \\
\hline $44 X-C C, 10-11$ & 397.13 & n.d. & 3.74 & 4.59 & 0.31 & 1.99 & 14.81 & 16 & 30 & 3.5 & 0.60 & 1.5 & 0.30 & 575 & 80 \\
\hline $45 X-1,29-30$ & 403.99 & n.d. & 1.00 & 2.28 & 0.24 & n.d. & 9.50 & 15 & 27 & 3.7 & 0.77 & 1.7 & 0.36 & 423 & 60 \\
\hline $46 X-1,40-42$ & 408.60 & 2.8 & 11.16 & 4.73 & 0.25 & 1.35 & 18.92 & 25 & 45 & 5.4 & 1.01 & 2.6 & 0.49 & 539 & 83 \\
\hline $46 X-1,140-150$ & 409.70 & n.d. & 8.08 & 1.39 & n.d. & 0.90 & n.d. & 17 & 30 & 3.8 & 0.74 & 2.1 & 0.41 & 448 & 70 \\
\hline $47 X-1,51-52$ & 418.21 & n.d. & 2.75 & 2.24 & 0.25 & 1.11 & 8.96 & 17 & 30 & 3.9 & 0.79 & 1.9 & 0.38 & 558 & 69 \\
\hline $48 X-C C, 18-19$ & 427.31 & n.d. & 3.49 & 2.33 & 0.26 & 1.14 & 8.97 & 16 & 26 & 3.7 & 0.80 & 1.8 & 0.37 & 497 & 63 \\
\hline
\end{tabular}


Table 2 (continued).

\begin{tabular}{|c|c|c|c|c|c|c|c|c|c|}
\hline Sample ID & $\begin{array}{l}\mathrm{Fe} \\
(\%)\end{array}$ & $\begin{array}{c}\mathrm{Cs} \\
\text { (ppm) }\end{array}$ & $\begin{array}{c}\text { Co } \\
\text { (ppm) }\end{array}$ & $\begin{array}{l}\text { Hf } \\
(\mathrm{ppm})\end{array}$ & $\begin{array}{c}\mathrm{Rb} \\
(\mathrm{ppm})\end{array}$ & $\underset{(\mathrm{ppm})}{\mathrm{Sc}}$ & $\begin{array}{c}\text { Th } \\
\text { (ppm) }\end{array}$ & $\begin{array}{c}\text { Sb } \\
(\mathrm{ppm})\end{array}$ & $\underset{\text { (ppm) }}{\mathrm{Br}}$ \\
\hline $01 \mathrm{H}-3,76-78$ & 3.36 & 9.5 & 8.0 & 6.0 & 99 & 12.88 & 9.32 & 2.3 & 135 \\
\hline $01 \mathrm{H}-6,56-58$ & 3.65 & 10.8 & 9.2 & 4.3 & 101 & 14.94 & 9.35 & 2.0 & 171 \\
\hline $02 \mathrm{H}-3,45-47$ & 2.64 & 8.2 & 7.3 & 3.0 & 80 & 10.92 & 6.45 & 1.6 & 180 \\
\hline $03 \mathrm{H}-2,55-57$ & 3.32 & 8.9 & 8.2 & 3.4 & 81 & 12.55 & 7.43 & 1.8 & 169 \\
\hline $03 \mathrm{H}-5,67-77$ & 3.71 & 10.0 & 8.9 & 3.3 & 95 & 14.05 & 8.73 & 3.0 & 154 \\
\hline $04 \mathrm{H}-1,127-128$ & 1.58 & 4.7 & 4.4 & 1.6 & 38 & 6.47 & 4.13 & 1.6 & 223 \\
\hline $05 \mathrm{H}-1,48-49$ & 1.81 & 6.2 & 4.9 & 1.9 & 55 & 7.20 & 4.83 & 1.4 & 184 \\
\hline $06 \mathrm{H}-3,140-150$ & 2.18 & 6.4 & 4.4 & 2.2 & 51 & 7.61 & 4.80 & 2.4 & 234 \\
\hline $07 \mathrm{H}-2,79-81$ & 3.09 & 9.5 & 7.2 & 2.9 & 85 & 11.40 & 7.35 & 3.1 & 168 \\
\hline $08 \mathrm{H}-1,46-48$ & 1.50 & 4.5 & 3.4 & 1.8 & 42 & 5.74 & 3.88 & 1.9 & 272 \\
\hline $09 \mathrm{H}-1,106-116$ & 1.80 & 4.9 & 3.7 & 1.4 & 42 & 6.40 & 3.36 & 1.6 & 208 \\
\hline $10 \mathrm{H}-1,94-96$ & 0.93 & 2.6 & 2.3 & 0.9 & 23 & 3.57 & 2.03 & 1.0 & 219 \\
\hline $12 X-1,140-150$ & 3.16 & 7.5 & 6.8 & 3.1 & 80 & 11.72 & 6.83 & 2.3 & 47 \\
\hline $14 X-C C, 2-3$ & 1.57 & 3.7 & 2.9 & 1.9 & 48 & 6.32 & 3.69 & 0.9 & 112 \\
\hline $15 X-1,134-139$ & 1.74 & 4.8 & 3.4 & 1.6 & 45 & 7.48 & 3.82 & 1.6 & 79 \\
\hline $17 \mathrm{H}-1,146-147$ & 2.00 & 4.7 & 3.3 & 2.6 & 58 & 8.94 & 4.84 & 1.2 & 54 \\
\hline $18 \mathrm{X}-1,145-150$ & 1.57 & 4.0 & 2.5 & 1.5 & 43 & 6.28 & 3.37 & 1.0 & 42 \\
\hline $18 X-2,84-86$ & 1.48 & 3.3 & 2.4 & 1.9 & 39 & 6.29 & 3.33 & 0.7 & 87 \\
\hline $20 X-1,59-61$ & 1.39 & 3.8 & 2.4 & 1.4 & 46 & 5.63 & 3.40 & 0.7 & 65 \\
\hline $20 X-2,44-46$ & 1.38 & 3.4 & 2.2 & 1.4 & 42 & 5.73 & 3.03 & 0.9 & 72 \\
\hline $21 X-1,93-95$ & 1.51 & 3.7 & 2.4 & 1.5 & 42 & 5.62 & 3.16 & 0.7 & 61 \\
\hline $22 X-2,63-64$ & 1.53 & 3.5 & 2.3 & 2.1 & 51 & 7.38 & 4.08 & 1.0 & 52 \\
\hline $24 X-1,117-120$ & 1.51 & 3.0 & 2.6 & 2.0 & 45 & 7.66 & 4.13 & 0.6 & 41 \\
\hline $25 \mathrm{X}-\mathrm{CC}, 18-20$ & 1.36 & 2.9 & 2.3 & 1.7 & 42 & 7.00 & 3.76 & 0.7 & 45 \\
\hline $26 X-C C, 11-13$ & 1.24 & 2.6 & 2.0 & 1.4 & 34 & 5.41 & 3.18 & 0.7 & 52 \\
\hline $27 X-1,106-113$ & 1.20 & 2.7 & 1.9 & 1.5 & 33 & 5.52 & 3.29 & 0.8 & 34 \\
\hline $28 \times-1,6-7$ & 1.01 & 2.0 & 1.7 & 1.6 & 30 & 4.98 & 3.08 & 0.6 & 55 \\
\hline $29 X-1,16-17$ & 0.76 & 1.7 & 1.9 & 0.7 & 20 & 3.13 & 1.70 & 0.9 & 66 \\
\hline $33 X-1,16-17$ & 2.86 & 5.9 & 6.8 & 2.2 & 67 & 10.77 & 5.11 & 1.9 & 37 \\
\hline $34 X-2,140-150$ & 4.93 & 8.1 & 9.2 & 3.0 & 99 & 17.32 & 6.61 & 2.9 & 38 \\
\hline $34 X-3,30-32$ & 4.24 & 8.8 & 9.7 & 3.1 & 103 & 15.60 & 6.86 & 3.2 & 47 \\
\hline $35 X-2,97-98$ & 4.47 & 6.5 & 10.1 & 3.1 & 82 & 16.11 & 6.07 & 1.6 & 33 \\
\hline $36 \mathrm{X}-1,80-85$ & 3.56 & 5.8 & 8.7 & 3.6 & 69 & 14.86 & 5.77 & 1.6 & 28 \\
\hline $37 X-2,83-84$ & 3.29 & 4.6 & 7.2 & 3.1 & 66 & 12.62 & 5.09 & 1.7 & 35 \\
\hline $39 X-2,131-141$ & 3.20 & 4.7 & 6.4 & 2.4 & 58 & 11.19 & 4.11 & 1.5 & 22 \\
\hline $40 X-2,9-11$ & 4.33 & 4.7 & 7.9 & 3.4 & 76 & 14.41 & 5.08 & 1.6 & 26 \\
\hline $41 X-C C, 10-11$ & 3.02 & 3.5 & 6.4 & 2.1 & 48 & 10.62 & 3.89 & 2.8 & 24 \\
\hline $42 X-C C, 12-13$ & 1.28 & 2.1 & 3.0 & 1.2 & 26 & 5.63 & 1.72 & 1.3 & 21 \\
\hline $44 X-C C, 10-11$ & 2.96 & 4.6 & 6.9 & 2.5 & 72 & 11.68 & 4.47 & 3.0 & 16 \\
\hline $45 X-1,29-30$ & 5.83 & 5.7 & 9.4 & 3.5 & 61 & 14.33 & 4.36 & 1.1 & 14 \\
\hline $46 X-1,40-42$ & 4.36 & 4.6 & 9.9 & 4.7 & 85 & 17.99 & 7.27 & 1.5 & 14 \\
\hline $46 \mathrm{X}-1,140-150$ & 2.70 & 3.1 & 6.4 & 2.9 & 56 & 12.64 & 4.60 & 1.6 & 22 \\
\hline $47 X-1,51-52$ & 3.86 & 4.5 & 8.4 & 3.7 & 73 & 16.01 & 4.96 & 0.9 & 17 \\
\hline $48 X-C C, 18-19$ & 3.43 & 4.3 & 8.5 & 3.6 & 66 & 17.27 & 4.50 & 0.8 & 22 \\
\hline
\end{tabular}


Table 3. Sediment chemistry Site 685 .

\begin{tabular}{|c|c|c|c|c|c|c|c|c|c|c|c|c|c|c|c|}
\hline Sample ID & $\begin{array}{l}\text { Depth } \\
\text { (mbsf) }\end{array}$ & $\begin{array}{c}\text { Opal-Si } \\
(\%)\end{array}$ & $\begin{array}{c}\mathrm{CaCO}_{3} \\
(\%)\end{array}$ & $\begin{array}{l}\text { TOC } \\
(\%)\end{array}$ & $\begin{array}{l}\text { TN } \\
(\%)\end{array}$ & $\begin{array}{l}\text { TRS } \\
(\%)\end{array}$ & $\mathrm{C} / \mathrm{N}$ & $\underset{(\mathrm{ppm})}{\mathrm{La}}$ & $\underset{(\mathrm{ppm})}{\mathrm{Ce}}$ & $\underset{(\mathrm{ppm})}{\mathrm{Sm}}$ & $\underset{(\mathrm{ppm})}{\mathrm{Eu}}$ & $\underset{(\mathrm{ppm})}{\mathrm{Yb}}$ & $\underset{(\mathrm{ppm})}{\mathrm{Lu}}$ & $\underset{(\mathrm{ppm})}{\mathrm{Ba}}$ & $\underset{(\mathrm{ppm})}{\mathrm{Cr}}$ \\
\hline $01 \mathrm{H}-2,40-41$ & 1.90 & n.d. & 0.58 & 7.72 & 1.76 & 1.13 & 4.4 & 25 & 45 & 5.3 & 1.06 & 1.8 & 0.46 & 874 & 84 \\
\hline $02 \mathrm{H}-3,44-45$ & 7.54 & 4.3 & 1.25 & 6.05 & 0.71 & 0.95 & 8.5 & 27 & 45 & 5.1 & 0.91 & 2.0 & 0.42 & 2002 & 77 \\
\hline $03 \mathrm{H}-3,35-36$ & 16.95 & n.d. & 3.25 & 7.41 & 0.92 & 1.05 & 8.1 & 26 & 45 & 5.1 & 0.96 & 2.1 & 0.41 & 702 & 74 \\
\hline $04 \mathrm{H}-2,19-20$ & 24.69 & n.d. & 1.09 & 4.73 & 0.86 & 0.87 & 5.5 & 22 & 38 & 4.5 & 0.83 & 1.8 & 0.49 & 1151 & 83 \\
\hline $05 \mathrm{H}-2,41-42$ & 34.51 & 4.1 & 0.75 & 5.63 & 0.78 & 1.08 & 7.2 & 29 & 50 & 5.5 & 1.04 & 2.3 & 0.51 & 1055 & 81 \\
\hline $06 \mathrm{H}-2,22-23$ & 43.82 & n.d. & 0.33 & 7.37 & 0.80 & 0.77 & 9.2 & 28 & 47 & 5.3 & 0.99 & 2.2 & 0.46 & 1227 & 87 \\
\hline $07 \mathrm{H}-2,33-34$ & 53.43 & n.d. & 1.33 & 6.74 & 0.82 & 0.89 & 8.2 & 24 & 40 & 4.7 & 0.95 & 2.3 & 0.47 & 929 & 86 \\
\hline $08 \mathrm{H}-3,55-56$ & 64.65 & n.d. & 1.42 & 4.94 & 1.30 & 0.37 & 3.8 & 26 & 43 & 5.1 & 0.95 & 2.4 & 0.53 & 1427 & 99 \\
\hline $09 \mathrm{H}-5,70-71$ & 76.17 & 6.4 & 5.25 & 8.08 & 0.78 & 2.20 & 10.4 & 22 & 36 & 3.9 & 0.72 & 2.2 & 0.42 & 876 & 77 \\
\hline $10 \mathrm{X}-2,30-31$ & 81.90 & n.d. & 1.75 & 5.89 & 0.76 & 0.52 & 7.7 & 23 & 39 & 4.2 & 0.78 & 2.3 & 0.41 & 919 & 87 \\
\hline $11 X-3,10-11$ & 92.70 & 7.9 & 4.92 & 9.09 & 0.97 & 0.37 & 9.4 & 20 & 32 & 3.5 & 0.69 & 1.7 & 0.36 & 935 & 76 \\
\hline $12 X-5,30-31$ & 105.40 & n.d. & 6.26 & 8.33 & 1.02 & 0.62 & 8.2 & 22 & 38 & 4.3 & 0.78 & 2.3 & 0.42 & 1036 & 78 \\
\hline $13 X-2,65-66$ & 110.75 & n.d. & 2.84 & 8.24 & 1.02 & 0.55 & 8.1 & 23 & 40 & 4.3 & 0.84 & 2.2 & 0.41 & 1029 & 77 \\
\hline $14 X-2,71-72$ & 120.31 & 4.7 & 2.00 & 7.35 & 0.80 & 1.07 & 9.2 & 27 & 46 & 5.2 & 0.94 & 2.5 & 0.44 & 1160 & 86 \\
\hline $15 X-3,47-48$ & 129.87 & n.d. & 2.00 & 2.94 & 0.30 & 1.21 & 9.8 & 18 & 30 & 3.4 & 0.59 & 1.6 & 0.32 & 610 & 61 \\
\hline $16 X-2,19-20$ & 138.79 & n.d. & 4.59 & 7.85 & 0.95 & 0.99 & 8.3 & 16 & 27 & 3.2 & 0.55 & 1.4 & 0.27 & 717 & 56 \\
\hline $17 X-4,45-46$ & 151.05 & 7.6 & 2.42 & 6.09 & 0.67 & 0.52 & 9.1 & 21 & 36 & 3.9 & 0.75 & 1.6 & 0.26 & 913 & 69 \\
\hline $18 \mathrm{X}-1,31-32$ & 156.41 & n.d. & 4.25 & 7.63 & 0.79 & n.d. & 9.7 & 25 & 44 & 4.5 & 0.88 & 2.0 & 0.38 & 827 & 68 \\
\hline $19 X-1,23-24$ & 165.83 & n.d. & 2.67 & 7.18 & 0.70 & 1.50 & 10.3 & 24 & 41 & 4.3 & 0.83 & 1.9 & 0.38 & 985 & 69 \\
\hline $20 X-4,72-73$ & 180.04 & 5.2 & 1.92 & 8.66 & 0.96 & 0.60 & 9.0 & 23 & 39 & 4.3 & 0.82 & 1.9 & 0.34 & 1124 & 72 \\
\hline $22 X-2,91-92$ & 196.51 & n.d. & 2.59 & 6.49 & 1.28 & 0.49 & 5.1 & 23 & 38 & 4.2 & 0.79 & 2.0 & 0.37 & 1035 & 86 \\
\hline $25 \mathrm{X}-\mathrm{CC}, 0-1$ & 223.00 & 4.3 & 1.33 & 10.07 & 1.01 & n.d. & 10.0 & 22 & 35 & 4.0 & 0.73 & 1.9 & 0.39 & 1079 & 138 \\
\hline $27 X-1,49-50$ & 234.09 & n.d. & 0.92 & 12.86 & 1.06 & n.d. & 12.1 & 19 & 28 & 3.4 & 0.58 & 1.5 & 0.36 & 1049 & 136 \\
\hline $28 X-3,48-49$ & 246.58 & 5.5 & 0.83 & 3.88 & 0.53 & 0.50 & 7.3 & 24 & 42 & 4.9 & 0.86 & 2.1 & 0.40 & 1007 & 138 \\
\hline $29 X-2,14-15$ & 254.24 & n.d. & 1.33 & 6.57 & 0.75 & n.d. & 8.8 & 19 & 28 & 3.4 & 0.66 & 1.8 & 0.35 & 1035 & 131 \\
\hline $30 X-2,49-50$ & 264.09 & n.d. & 1.42 & 4.88 & 0.60 & 1.00 & 8.1 & 19 & 28 & 3.4 & 0.59 & 1.9 & 0.29 & 917 & 105 \\
\hline $34 X-2,26-27$ & 301.86 & 7.0 & 0.75 & 2.90 & 0.88 & n.d. & 3.3 & 19 & 32 & 3.6 & 0.66 & 1.5 & 0.27 & 876 & 78 \\
\hline $35 X-6,29-30$ & 316.13 & n.d. & 8.99 & 2.84 & 0.94 & 1.44 & 3.0 & 12 & 20 & 2.2 & 0.48 & 1.1 & 0.27 & 904 & 87 \\
\hline $36 X-3,140-150$ & 326.90 & n.d. & 0.29 & 2.40 & n.d. & n.d. & n.d. & 25 & 42 & 4.9 & 0.91 & 2.8 & 0.47 & 862 & 158 \\
\hline $36 X-7,40-41$ & 327.40 & n.d. & 0.58 & 4.67 & 1.08 & n.d. & 4.3 & 10 & 14 & 1.6 & 0.30 & 0.7 & 0.19 & 644 & 58 \\
\hline $37 X-1,146-147$ & 330.06 & 7.9 & 1.00 & 6.60 & 0.59 & 1.20 & 11.2 & 16 & 25 & 2.9 & 0.58 & 1.4 & 0.32 & 882 & 89 \\
\hline $38 \mathrm{X}-\mathrm{CC}, 20-21$ & 338.82 & n.d. & 42.10 & 12.45 & 0.79 & 0.73 & 15.8 & 17 & 26 & 2.9 & 0.52 & 2.0 & 0.45 & 1108 & 112 \\
\hline $39 X-3,140-150$ & 352.00 & n.d. & 0.25 & 1.72 & n.d. & n.d. & n.d. & 24 & 41 & 4.4 & 0.79 & 1.9 & 0.37 & 1100 & 136 \\
\hline $40 X-1,1-2$ & 357.11 & 6.7 & 1.50 & 5.09 & 0.52 & 0.68 & 9.8 & 19 & 30 & 3.4 & 0.73 & 1.9 & 0.42 & 941 & 126 \\
\hline $42 X-C C, 9-10$ & 378.79 & n.d. & 2.00 & 4.83 & 0.56 & 1.56 & 8.6 & 20 & 33 & 3.7 & 0.74 & 1.9 & 0.45 & 864 & 139 \\
\hline $43 X-1,30-31$ & 385.90 & 8.2 & 0.42 & 4.70 & 0.79 & 0.77 & 5.9 & 7 & 10 & 1.7 & 0.33 & 0.9 & 0.18 & 401 & 62 \\
\hline $44 X-3,40-50$ & 396.00 & n.d. & 6.25 & 1.61 & n.d. & n.d. & n.d. & 19 & 34 & 4.2 & 0.74 & 2.2 & 0.39 & 920 & 123 \\
\hline $47 X-1,39-40$ & 421.49 & 3.5 & 3.58 & 3.80 & 0.96 & n.d. & 4.0 & 14 & 24 & 2.6 & 0.48 & 1.0 & 0.24 & 519 & 57 \\
\hline $48 X-1,61-62$ & 431.21 & n.d. & 4.75 & 5.09 & 0.93 & 0.49 & 5.5 & 21 & 36 & 3.9 & 0.73 & 1.8 & 0.36 & 696 & 123 \\
\hline 49X-CC, 9-10 & 440.59 & n.d. & 4.75 & 4.95 & 0.44 & n.d. & 11.2 & 20 & 36 & 3.7 & 0.69 & 1.8 & 0.33 & 662 & 126 \\
\hline $50 X-1,52-53$ & 450.12 & 6.5 & 4.75 & 5.30 & 0.83 & n.d. & 6.4 & 18 & 30 & 3.9 & 0.72 & 1.8 & 0.34 & 728 & 122 \\
\hline $50 \mathrm{X}-1,92-102$ & 450.52 & n.d. & 3.25 & 2.33 & n.d. & 1.31 & n.d. & 15 & 27 & 3.6 & 0.65 & 2.0 & 0.38 & 621 & 112 \\
\hline
\end{tabular}


Table 3 (continued).

\begin{tabular}{|c|c|c|c|c|c|c|c|c|c|}
\hline Sample ID & $\begin{array}{l}\mathrm{Fe} \\
(\%)\end{array}$ & $\underset{(\mathrm{ppm})}{\mathrm{Cs}}$ & $\begin{array}{c}\text { Co } \\
\text { (ppm) }\end{array}$ & $\underset{(\mathrm{ppm})}{\mathrm{Hf}}$ & $\begin{array}{c}\mathbf{R b} \\
(\mathrm{ppm})\end{array}$ & $\underset{(\mathrm{ppm})}{\mathrm{Sc}}$ & $\begin{array}{l}\text { Th } \\
\text { (ppm) }\end{array}$ & $\begin{array}{c}\mathrm{Sb} \\
(\mathrm{ppm})\end{array}$ & $\begin{array}{c}\mathrm{Br} \\
(\mathrm{ppm})\end{array}$ \\
\hline $01 \mathrm{H}-2,40-41$ & 3.84 & 10.5 & 10.1 & 3.2 & 99 & 14.0 & 8.7 & 2.5 & 213 \\
\hline $02 \mathrm{H}-3,44-45$ & 3.58 & 10.9 & 8.6 & 3.7 & 95 & 12.9 & 9.1 & 2.3 & 131 \\
\hline $03 \mathrm{H}-3,35-36$ & 3.37 & 9.4 & 8.1 & 3.7 & 91 & 11.6 & 8.4 & 2.1 & 181 \\
\hline $04 \mathrm{H}-2,19-20$ & 3.45 & 7.3 & 9.1 & 3.0 & 75 & 14.2 & 6.7 & 2.2 & 139 \\
\hline $05 \mathrm{H}-2,41-42$ & 3.77 & 11.9 & 9.2 & 3.9 & 110 & 14.5 & 9.6 & 2.4 & 127 \\
\hline $06 \mathrm{H}-2,22-23$ & 3.59 & 9.8 & 8.4 & 3.7 & 92 & 14.1 & 8.6 & 2.1 & 200 \\
\hline $07 \mathrm{H}-2,33-34$ & 3.74 & 9.2 & 8.9 & 3.3 & 87 & 13.8 & 7.7 & 1.7 & 163 \\
\hline $08 \mathrm{H}-3,55-56$ & 3.67 & 9.1 & 7.5 & 3.9 & 84 & 14.5 & 8.1 & 1.7 & 131 \\
\hline $09 \mathrm{H}-5,70-71$ & 4.45 & 8.6 & 7.1 & 3.1 & 67 & 13.0 & 6.9 & 1.6 & 148 \\
\hline $10 X-2,30-31$ & 3.63 & 10.7 & 9.4 & 3.5 & 82 & 13.7 & 7.7 & 1.6 & 128 \\
\hline $11 X-3,10-11$ & 2.76 & 8.9 & 6.3 & 2.8 & 64 & 10.8 & 6.0 & 1.5 & 196 \\
\hline $12 X-5,30-31$ & 3.26 & 9.6 & 7.6 & 3.3 & 68 & 12.2 & 7.0 & 1.7 & 178 \\
\hline $13 X-2,65-66$ & 3.39 & 11.4 & 8.4 & 3.4 & 86 & 12.8 & 7.6 & 2.0 & 155 \\
\hline $14 X-2,71-72$ & 3.62 & 12.4 & 8.6 & 3.9 & 85 & 13.8 & 8.8 & 2.0 & 136 \\
\hline $15 X-3,47-48$ & 2.37 & 8.7 & 5.6 & 2.7 & 57 & 9.2 & 5.4 & 1.3 & 211 \\
\hline $16 \times-2,19-20$ & 2.23 & 7.2 & 5.0 & 2.3 & 53 & 8.3 & 4.8 & 1.4 & 199 \\
\hline $17 X-4,45-46$ & 2.90 & 8.4 & 7.2 & 3.1 & 66 & 11.3 & 6.6 & 1.9 & 135 \\
\hline $18 \mathrm{X}-1,31-32$ & 2.88 & 10.8 & 6.8 & 3.5 & 95 & 11.5 & 7.8 & 2.3 & 148 \\
\hline $19 X-1,23-24$ & 2.71 & 10.1 & 6.7 & 3.7 & 76 & 11.3 & 7.5 & 2.1 & 107 \\
\hline $20 \times-4,72-73$ & 2.79 & 9.3 & 6.2 & 3.2 & 73 & 11.0 & 7.1 & 2.3 & 127 \\
\hline $22 \mathrm{X}-2,91-92$ & 3.21 & 8.5 & 6.8 & 3.2 & 71 & 12.1 & 6.9 & 2.0 & 111 \\
\hline $25 \mathrm{X}-\mathrm{CC}, 0-1$ & 2.63 & 7.7 & 6.5 & 2.9 & 81 & 11.3 & 6.2 & 1.9 & 142 \\
\hline $27 X-1,49-50$ & 2.86 & 7.2 & 6.4 & 2.5 & 70 & 10.6 & 5.2 & 1.8 & 136 \\
\hline $28 X-3,48-49$ & 2.95 & 8.0 & 7.3 & 3.2 & 75 & 13.9 & 7.1 & 2.1 & 45 \\
\hline $29 X-2,14-15$ & 3.03 & 7.1 & 7.2 & 2.8 & 80 & 12.1 & 5.7 & 1.6. & 86 \\
\hline $30 X-2,49-50$ & 3.10 & 6.7 & 7.4 & 2.5 & 60 & 11.0 & 5.9 & 1.7 & 63 \\
\hline $34 X-2,26-27$ & 2.57 & 6.8 & 6.0 & 3.0 & 77 & 8.5 & 6.6 & 2.6 & 44 \\
\hline $35 \mathrm{X}-6,29-30$ & 2.79 & 4.7 & 5.4 & 1.8 & 62 & 7.7 & 3.9 & 1.9 & 73 \\
\hline $36 \mathrm{X}-3,140-150$ & 4.44 & 9.7 & 9.2 & 3.4 & 93 & 17.0 & 8.1 & 1.6 & 42 \\
\hline $36 X-7,40-41$ & 1.89 & 2.9 & 3.9 & 1.1 & 40 & 5.1 & 2.6 & 1.3 & 67 \\
\hline $37 X-1,146-147$ & 2.75 & 5.3 & 5.6 & 2.2 & 70 & 9.4 & 4.8 & 1.5 & 52 \\
\hline $38 X-C C, 20-21$ & 5.32 & 5.2 & 5.5 & 2.2 & 73 & 12.5 & 4.7 & 1.9 & 70 \\
\hline $39 X-3,140-150$ & 2.79 & 7.9 & 6.4 & 3.2 & 84 & 10.3 & 7.6 & 1.3 & 51 \\
\hline $40 X-1,1-2$ & 3.06 & 7.4 & 6.7 & 2.8 & 91 & 12.3 & 6.1 & 1.9 & 49 \\
\hline $42 X-C C, 9-10$ & 4.03 & 7.5 & 7.5 & 2.8 & 90 & 12.5 & 6.5 & 2.0 & 39 \\
\hline $43 X-1,30-31$ & 1.37 & 1.8 & 2.9 & 1.1 & 28 & 5.8 & 1.6 & 1.0 & 67 \\
\hline $44 X-3,40-50$ & 4.06 & 7.2 & 9.1 & 3.1 & 76 & 15.3 & 6.2 & 1.8 & 43 \\
\hline $47 X-1,39-40$ & 2.32 & 4.0 & 4.6 & 2.1 & 55 & 7.6 & 4.0 & 1.3 & 36 \\
\hline $48 X-1,61-62$ & 3.98 & 7.3 & 7.1 & 2.8 & 90 & 13.1 & 6.7 & 1.6 & 78 \\
\hline $49 X-C C, 9-10$ & 3.56 & 7.1 & 7.3 & 2.8 & 86 & 12.6 & 6.5 & 1.5 & 91 \\
\hline $50 \times-1,52-53$ & 3.60 & 5.1 & 7.7 & 2.9 & 74 & 13.9 & 5.3 & 1.9 & 60 \\
\hline $50 \mathrm{X}-1,92-102$ & 3.28 & 4.8 & 7.2 & 2.6 & 53 & 12.6 & 4.7 & 1.9 & 68 \\
\hline
\end{tabular}


Table 4. Sediment chemistry at Site 688.

\begin{tabular}{|c|c|c|c|c|c|c|c|c|c|c|c|c|}
\hline $\begin{array}{l}\text { Core, section, } \\
\text { interval }(\mathrm{cm})\end{array}$ & $\begin{array}{l}\text { Depth } \\
\text { (mbsf) }\end{array}$ & Opal-Si & $\begin{array}{c}\mathrm{CaCO}_{3} \\
(\%)\end{array}$ & $\begin{array}{l}\mathrm{T}_{\max } \\
\left({ }^{\circ} \mathrm{C}\right)\end{array}$ & $\begin{array}{c}\mathrm{S}_{1} \\
(\mathrm{mg} / \mathrm{g})\end{array}$ & $\underset{(\mathrm{mg} / \mathrm{g})}{\mathrm{S}_{2}}$ & $\begin{array}{c}\mathrm{S}_{3} \\
(\mathrm{mg} / \mathrm{g})\end{array}$ & $\begin{array}{l}\text { TOC } \\
(\%)\end{array}$ & $\begin{array}{l}\text { TRS } \\
(\%)\end{array}$ & $\underset{(\mathrm{ppm})}{\mathrm{La}}$ & $\underset{(\mathrm{ppm})}{\mathrm{Ce}}$ & $\underset{(\mathrm{ppm})}{\mathrm{Sm}}$ \\
\hline $688 \mathrm{~A}-01 \mathrm{H}-5,82-83$ & 6.8 & n.d. & 1.33 & n.d. & n.d. & n.d. & n.d. & 3.02 & 0.66 & 30 & 48 & 6.1 \\
\hline $02 \mathrm{H}-1,44-45$ & 8.7 & 3.5 & 1.00 & n.d. & n.d. & n.d. & n.d. & 2.77 & 1.00 & 28 & 43 & 5.7 \\
\hline $02 \mathrm{H}-3,44-45$ & 11.7 & n.d. & 1.33 & n.d. & n.d. & n.d. & n.d. & 1.85 & 0.39 & 29 & 48 & 6.1 \\
\hline $02 \mathrm{H}-5,44-45$ & 14.7 & n.d. & 1.75 & 423 & 1.15 & 4.48 & 1.97 & 1.56 & 0.56 & 30 & 48 & 6.2 \\
\hline $02 \mathrm{H}-7,44-45$ & 17.7 & n.d. & 10.34 & 418 & 2.59 & 13.58 & 5.32 & 3.73 & 0.87 & 24 & 43 & 4.9 \\
\hline $03 \mathrm{H}-2,42-43$ & 19.7 & 6.6 & 10.68 & 406 & 3.24 & 10.77 & 4.59 & 3.28 & 116 & 23 & 40 & 4.7 \\
\hline $03 \mathrm{H}-4,42-43$ & 22.7 & n.d. & 10.89 & 422 & 1.79 & 9.10 & 4.51 & 2.72 & 118 & 25 & 44 & 5.1 \\
\hline $03 \mathrm{H}-4,140-150$ & 23.7 & n.d. & n.d. & 424 & 0.68 & 5.07 & 2.58 & 1.99 & 1.14 & n.d. & n.d. & n.d. \\
\hline $04 \mathrm{H}-3,44-45$ & 30.7 & n.d. & 6.51 & n.d. & n.d. & n.d. & n.d. & 2.70 & 0.88 & 31 & 51 & 6.4 \\
\hline $05 \mathrm{H}-3,64-65$ & 40.4 & 4.8 & 49.87 & 423 & 0.90 & 5.17 & 2.49 & 1.55 & 0.84 & 31 & 55 & 6.0 \\
\hline $06 \mathrm{H}-2,63-64$ & 48.5 & n.d. & 13.76 & 422 & 1.20 & 5.87 & 3.13 & 1.91 & 0.93 & 25 & 44 & 5.5 \\
\hline $06 \mathrm{H}-3,145-150$ & 50.8 & n.d. & 8.48 & 424 & 0.68 & 5.07 & 2.58 & 2.14 & 0.81 & 26 & 46 & 5.6 \\
\hline $08 \mathrm{X}-1,57-58$ & 65.9 & 3.2 & 21.35 & n.d. & n.d. & n.d. & n.d. & 3.67 & 1.08 & 25 & 42 & 5.3 \\
\hline $09 X-5,100-111$ & 81.9 & n.d. & 9.17 & 413 & 3.50 & 16.41 & 5.12 & 3.87 & 1.06 & 25 & 47 & 5.1 \\
\hline $09 X-5,140-150$ & 82.2 & n.d. & n.d. & 418 & 2.16 & 16.37 & 3.94 & 4.73 & 1.34 & n.d. & n.d. & n.d. \\
\hline $09 X-6,110-111$ & 83.4 & n.d. & 1.50 & 405 & 2.98 & 12.39 & 5.20 & 3.32 & 0.80 & 23 & 45 & 4.7 \\
\hline $10 \mathrm{X}-2,32-33$ & 86.1 & 4.5 & 8.76 & 404 & 2.28 & 8.73 & 6.14 & 3.55 & 1.32 & 24 & 44 & 4.7 \\
\hline $11 X-2,28-29$ & 95.6 & n.d. & 8.09 & n.d. & n.d. & n.d. & n.d. & 3.76 & 0.86 & 27 & 49 & 5.3 \\
\hline $12 X-1,55-56$ & 103.9 & 2.8 & 8.59 & 414 & 3.50 & 17.37 & 6.04 & 4.24 & 0.68 & 25 & 43 & 4.7 \\
\hline $13 X-2,66-67$ & 115.0 & n.d. & 5.34 & 420 & 2.28 & 13.60 & 4.77 & 3.67 & 0.55 & 29 & 51 & 5.6 \\
\hline $13 X-6,66-67$ & 121.0 & n.d. & 11.84 & 419 & 2.29 & 12.94 & 5.41 & 3.56 & 0.73 & 27 & & \\
\hline $14 X-2,85-86$ & 124.7 & n.d. & 8.84 & 414 & 2.46 & 12.79 & 7.37 & 3.76 & 0.73 & 27 & 48 & 5.3 \\
\hline $14 X-6,85-86$ & 130.7 & 2.8 & 4.34 & 417 & 2.82 & 15.56 & 6.24 & 4.08 & 0.32 & 28 & 47 & 5.1 \\
\hline $16 \mathrm{X}-2,11-12$ & 142.9 & n.d. & 0.32 & 413 & 3.18 & 15.23 & 5.83 & 4.40 & 0.68 & 23 & 35 & 4.4 \\
\hline $16 X-6,12-13$ & 148.9 & n.d. & 2.50 & 419 & 3.48 & 19.87 & 5.94 & 4.87 & 0.52 & 26 & 42 & 5.2 \\
\hline $17 X-1,58-59$ & 151.4 & n.d. & 3.50 & 416 & 2.50 & 13.38 & 5.95 & 3.36 & 0.79 & 27 & 44 & 5.1 \\
\hline $17 X-6,58-59$ & 158.9 & n.d. & 2.75 & 421 & 2.24 & 13.88 & 4.79 & 3.53 & 0.52 & 28 & 46 & 5.1 \\
\hline $18 \mathrm{X}-1,133-135$ & 161.6 & 5.6 & 4.17 & 413 & 3.88 & 18.65 & 6.30 & 4.78 & 0.67 & 25 & 39 & 4.9 \\
\hline $19 X-1,58-59$ & 170.4 & n.d. & 8.59 & 419 & 2.27 & 14.67 & 5.71 & 3.71 & n.d. & 27 & 45 & 5.2 \\
\hline $19 X-3,140-150$ & 174.2 & n.d. & n.d. & 424 & 0.88 & 10.35 & 3.51 & 3.24 & 0.48 & n.d. & n.d. & n.d. \\
\hline $19 X-5,58-59$ & 176.4 & n.d. & 7.26 & 416 & 1.49 & 9.91 & 4.65 & 2.80 & 0.90 & 29 & 52 & 5.7 \\
\hline $21 X-1,63-64$ & 189.4 & n.d. & 14.68 & 409 & 2.47 & 12.21 & 6.86 & 3.53 & 0.68 & 23 & 37 & 4.4 \\
\hline $21 X-3,63-64$ & 192.4 & n.d. & 8.17 & 410 & 1.90 & 9.86 & 5.27 & 3.09 & 1.00 & 24 & 43 & 4.7 \\
\hline $21 X-3,108-118$ & 192.9 & n.d. & n.d. & 417 & 1.05 & 9.86 & 3.33 & 3.41 & 1.01 & n.d. & n.d. & n.d. \\
\hline $22 \mathrm{X}-1,20-21$ & 198.5 & 4.6 & 9.67 & 415 & 4.43 & 24.28 & 7.19 & 5.75 & 0.89 & 25 & 42 & 5.1 \\
\hline $23 X-1,69-70$ & 208.5 & n.d. & 2.92 & 418 & 2.36 & 14.34 & 4.72 & 3.38 & 0.69 & 23 & 40 & 4.8 \\
\hline $24 X-1,50-51$ & 217.8 & n.d. & 2.00 & 416 & 3.45 & 19.57 & 4.99 & 4.79 & 1.06 & 24 & 43 & 4.8 \\
\hline $25 \mathrm{X}-1,62-63$ & 227.4 & n.d. & 1.83 & 408 & 7.74 & 35.38 & 7.38 & 8.00 & 0.95 & 21 & 34 & 4.2 \\
\hline $26 \mathrm{X}-1,33-34$ & 236.6 & 5.3 & 1.42 & 409 & 4.20 & 21.03 & 5.21 & 5.17 & 0.85 & 24 & 43 & 4.6 \\
\hline $27 X-1,25-26$ & 246.1 & n.d. & 1.08 & 414 & 2.57 & 12.73 & 3.71 & 3.24 & 1.04 & 27 & 47 & 5.8 \\
\hline $27 X-4,140-150$ & 251.7 & n.d. & n.d. & 413 & 1.36 & 16.66 & 3.27 & 2.71 & 1.60 & n.d. & n.d. & n.d. \\
\hline $28 \mathrm{X}-1,55-56$ & 255.9 & n.d. & 1.92 & 423 & 2.36 & 17.36 & 7.00 & 4.99 & 1.26 & 27 & 46 & 5.6 \\
\hline $29 \times-2,38-39$ & 269.7 & n.d. & 1.10 & 410 & 4.15 & 23.64 & 4.91 & 5.44 & 1.00 & 26 & 47 & 5.7 \\
\hline $30 \times-1,39-40$ & 277.8 & 4.8 & 1.85 & 410 & 3.61 & 18.68 & 5.01 & 4.78 & 1.14 & 27 & 48 & 5.4 \\
\hline $31 X-1,39-40$ & 287.4 & n.d. & n.d. & n.d. & n.d. & n.d. & n.d. & 4.49 & 0.75 & 24 & 43 & 5.0 \\
\hline $32 \times-1,37-38$ & 297.0 & n.d. & 3.76 & 417 & 2.10 & 13.20 & 4.90 & 3.38 & 0.95 & 28 & 54 & 5.7 \\
\hline $33 X-1,86-87$ & 303.7 & n.d. & 3.43 & 415 & 2.91 & 18.68 & 5.58 & 4.64 & 0.71 & 23 & 40 & 4.6 \\
\hline $34 X-1,68-69$ & 313.0 & 4.2 & 1.75 & 407 & 4.17 & 20.46 & 4.25 & 4.51 & 0.27 & 26 & 46 & 5.1 \\
\hline $36 \mathrm{X}-1,85-86$ & 332.2 & n.d. & 1.75 & 416 & 2.69 & 15.85 & 3.50 & 3.75 & 1.12 & 26 & 47 & 5.9 \\
\hline $36 X-6,140-150$ & 339.9 & n.d. & n.d. & 427 & 1.15 & 15.81 & 2.78 & 3.72 & 1.04 & n.d. & n.d. & n.d. \\
\hline $37 X-2,59-61$ & 342.9 & n.d. & 1.00 & 402 & 7.07 & 31.54 & 5.93 & 6.93 & 0.11 & 21 & 37 & 4.6 \\
\hline
\end{tabular}


Table 4 (continued).

\begin{tabular}{|c|c|c|c|c|c|c|c|c|c|c|c|c|c|}
\hline$\underset{(\mathrm{ppm})}{\mathrm{Eu}}$ & $\underset{(\mathrm{ppm})}{\mathrm{Yb}}$ & $\underset{(\mathrm{ppm})}{\mathrm{Lu}}$ & $\underset{(\mathrm{ppm})}{\mathrm{Ba}}$ & $\underset{(\mathrm{ppm})}{\mathrm{Cr}}$ & $\begin{array}{l}\mathrm{Fe} \\
(\%)\end{array}$ & $\underset{(\mathrm{ppm})}{\mathrm{Cs}}$ & $\underset{(\mathrm{ppm})}{\mathrm{Co}}$ & $\underset{(\mathrm{ppm})}{\mathrm{Hf}}$ & $\begin{array}{c}\mathrm{Rb} \\
(\mathrm{ppm})\end{array}$ & $\underset{(\mathrm{ppm})}{\mathrm{Sc}}$ & $\underset{(\mathrm{ppm})}{\mathrm{Th}}$ & $\underset{(\mathrm{ppm})}{\mathrm{Sb}}$ & $\begin{array}{c}\mathrm{Br} \\
\text { (ppm) }\end{array}$ \\
\hline 1.30 & 3.39 & 0.72 & 1819 & 92 & 3.58 & 13.2 & 9.6 & 3.8 & 117 & 15.0 & 8.9 & 2.5 & 211 \\
\hline 1.14 & 3.17 & 0.66 & 2060 & 102 & 4.03 & 11.6 & 9.3 & 3.4 & 111 & 15.3 & 8.3 & 0.9 & 185 \\
\hline 1.14 & 3.44 & 0.64 & 2576 & 94 & 2.96 & 10.5 & 7.9 & 3.7 & 93 & 14.3 & 8.7 & 2.1 & 175 \\
\hline 1.07 & 3.83 & 0.75 & 3310 & 91 & 3.22 & 9.0 & 8.0 & 4.4 & 99 & 13.4 & 8.8 & 2.0 & 117 \\
\hline 1.01 & 2.47 & 0.47 & 868 & 127 & 3.69 & 43.4 & 9.5 & 3.3 & 114 & 14.3 & 8.5 & 2.7 & 256 \\
\hline 1.03 & 2.44 & 0.47 & 944 & 88 & 3.36 & 12.3 & 7.8 & 3.1 & 98 & 11.7 & 7.4 & 2.6 & 222 \\
\hline 1.04 & 2.46 & 0.46 & 1050 & 73 & 3.27 & 12.5 & 7.8 & 3.5 & 100 & 11.6 & 8.0 & 1.8 & 180 \\
\hline n.d. & n.d. & n.d. & n.d. & n.d. & n.d. & n.d. & n.d. & n.d. & n.d. & n.d. & n.d. & n.d. & n.d. \\
\hline 1.09 & 2.93 & 0.64 & 1754 & 83 & 3.49 & 13.8 & 9.2 & 4.2 & 124 & 14.5 & 9.4 & 2.5 & 170 \\
\hline 1.32 & 2.57 & 0.57 & 4684 & 84 & 4.19 & 15.1 & 10.1 & 4.3 & 106 & 14.3 & 10.1 & 3.5 & 190 \\
\hline 0.93 & 2.56 & 0.63 & 1376 & 80 & 3.41 & 12.1 & 8.7 & 3.6 & 97 & 12.9 & 8.3 & 2.6 & 150 \\
\hline 1.09 & 3.20 & 0.66 & 1321 & 89 & 3.19 & 10.5 & 8.3 & 3.9 & 89 & 13.7 & 8.2 & 2.2 & 70 \\
\hline 0.89 & 2.98 & 0.62 & 1509 & 92 & 3.19 & 12.4 & 8.4 & 3.4 & 91 & 13.1 & 7.6 & 2.6 & 230 \\
\hline 1.03 & 2.62 & 0.53 & 1006 & 107 & 3.08 & 13.4 & 8.1 & 3.9 & 93 & 12.7 & 8.7 & 2.4 & 278 \\
\hline n.d. & n.d. & n.d. & n.d. & n.d. & n.d. & n.d. & n.d. & n.d. & n.d. & n.d. & n.d. & n.d. & n.d. \\
\hline 0.96 & 2.21 & 0.46 & 986 & 86 & 2.70 & 13.3 & 6.9 & 3.5 & 91 & 12.4 & 8.1 & 2.6 & 184 \\
\hline 0.88 & 2.17 & 0.41 & 1036 & 75 & 3.66 & 12.8 & 7.7 & 3.5 & 94 & 11.1 & 8.2 & 2.6 & 187 \\
\hline 0.90 & 2.64 & 0.53 & 12 & 90 & 3.23 & 14.0 & 8.4 & 3.9 & 107 & 13.9 & 9.1 & 2.4 & 217 \\
\hline 0.92 & 2.60 & 0.50 & 1404 & 98 & 2.95 & 13.2 & 7.3 & 3.5 & 96 & 11.6 & 7.7 & 2.3 & 275 \\
\hline 1.06 & 2.75 & 0.51 & 1441 & 100 & 3.58 & 15.3 & 9.2 & 4.5 & 112 & 13.4 & 9.9 & 3.0 & 239 \\
\hline 1.01 & 2.71 & 0.52 & 1348 & 84 & 3.54 & 14.3 & 8.9 & 3.8 & 112 & 13.7 & 9.3 & 2.5 & 230 \\
\hline 0.95 & 2.93 & 0.56 & 1590 & 97 & 4.17 & 15.6 & 9.3 & 3.9 & 101 & 15.1 & 9.2 & 2.6 & 242 \\
\hline 1.02 & 2.93 & 0.54 & 1422 & 101 & 3.35 & 12.8 & 8.0 & 3.8 & 100 & 13.3 & 8.7 & 2.3 & 264 \\
\hline 0.80 & 2.63 & 0.51 & 1957 & 101 & 3.12 & 10.3 & 7.3 & 2.9 & 79 & 11.9 & 6.6 & 2.3 & 264 \\
\hline 0.90 & 2.98 & 0.60 & 1899 & 117 & 3.30 & 11.6 & 8.4 & 3.5 & 89 & 13.7 & 8.0 & 2.7 & 287 \\
\hline 0.92 & 3.10 & 0.56 & 1729 & 108 & 3.79 & 12.0 & 8.3 & 3.7 & 103 & 14.2 & 8.5 & 2.5 & 237 \\
\hline 0.98 & 3.17 & 0.53 & 1883 & 115 & 3.48 & 12.1 & 8.3 & 3.6 & 100 & 13.9 & 8.5 & 2.5 & 249 \\
\hline 0.81 & 2.99 & 0.54 & 1857 & 107 & 3.10 & 10.3 & 7.6 & 3.6 & 88 & 12.1 & 7.6 & 2.3 & 301 \\
\hline 0.91 & 3.01 & 0.54 & 1406 & 113 & 3.88 & 12.5 & 9.2 & 3.9 & 112 & 14.8 & 9.0 & 2.3 & 225 \\
\hline n.d. & n.d. & n.d. & n.d. & n.d. & n.d. & n.d. & n.d. & n.d. & n.d. & n.d. & n.d. & n.d. & n.d. \\
\hline 1.11 & 3.06 & 0.56 & 1270 & 87 & 4.12 & 14.7 & 9.7 & 4.2 & 118 & 14.7 & 10.2 & 3.1 & 163 \\
\hline 0.77 & 2.19 & 0.46 & 1452 & 100 & 3.35 & 10.7 & 4.1 & 3.2 & 82 & 13.5 & 7.3 & 1.9 & 210 \\
\hline 0.76 & 2.59 & 0.48 & 1265 & 101 & 3.63 & 11.9 & 8.3 & 3.5 & 92 & 13.3 & 8.5 & 2.0 & 219 \\
\hline n.d. & n.d. & n.d. & n.d. & n.d. & n.d. & n.d. & n.d. & n.d. & n.d. & n.d. & n.d. & n.d. & n.d. \\
\hline 0.89 & 3.11 & 0.62 & 1472 & 110 & 3.16 & 11.0 & 7.6 & 3.4 & 85 & 13.2 & 7.7 & 2.6 & 279 \\
\hline 0.83 & 2.70 & 0.53 & 1518 & 102 & 3.05 & 9.1 & 7.0 & 3.0 & 78 & 11.7 & 7.1 & 2.0 & 234 \\
\hline 0.81 & 2.49 & 0.49 & 1511 & 128 & 3.33 & 11.3 & 9.1 & 3.3 & 92 & 13.6 & 8.2 & 2.2 & 262 \\
\hline 0.66 & 2.18 & 0.46 & 1650 & 123 & 2.56 & 8.8 & 6.3 & 3.0 & 70 & 10.1 & 6.2 & 1.9 & 301 \\
\hline 0.78 & 2.11 & 0.45 & 1589 & 103 & 2.91 & $\begin{array}{r}0.0 \\
11.7\end{array}$ & 7.2 & 3.6 & 86 & 11.4 & 7.9 & 2.4 & 234 \\
\hline 0.92 & 3.02 & 0.64 & 1808 & 149 & 3.55 & 10.1 & 8.4 & 3.8 & 90 & 14.2 & 8.2 & 2.3 & 179 \\
\hline n.d. & n.d. & n.d. & n.d. & n.d. & n.d. & n.d. & n.d. & n.d. & n.d. & n.d. & n.d. & n.d. & n.d. \\
\hline 0.93 & 2.78 & 0.52 & 1083 & 103 & 3.12 & 12.0 & 7.9 & 3.6 & 96 & 12.0 & 8.3 & 2.2 & 221 \\
\hline 0.99 & 2.79 & 0.63 & 1294 & 137 & 3.02 & 12.8 & 8.1 & 3.3 & 88 & 13.6 & 8.6 & 2.8 & 247 \\
\hline 0.97 & 2.48 & 0.57 & 1311 & 109 & 3.45 & 13.2 & 9.5 & 3.6 & 92 & 12.9 & 8.9 & 2.7 & 204 \\
\hline 1.07 & 2.56 & 0.54 & 1518 & 127 & 3.03 & 10.9 & 7.7 & 3.3 & 86 & 12.6 & 7.9 & 2.6 & 201 \\
\hline 0.98 & 2.57 & 0.57 & 1180 & 120 & 4.13 & 13.7 & 9.9 & 3.9 & 96 & 15.8 & 9.6 & 2.6 & 166 \\
\hline 0.74 & 2.39 & 0.49 & 1258 & 103 & 2.74 & 11.1 & 7.8 & 3.1 & 77 & 10.9 & 7.8 & 1.9 & 170 \\
\hline 0.88 & 2.60 & 0.52 & 1858 & 133 & 3.51 & 12.8 & 8.6 & 3.5 & 97 & 13.4 & 8.5 & 2.8 & 187 \\
\hline 0.87 & 2.77 & 0.68 & 1770 & 146 & 3.73 & 13.0 & $\begin{array}{l}0.0 \\
9.9\end{array}$ & 3.7 & 87 & 14.5 & 8.6 & 2.5 & 366 \\
\hline n.d. & n.d. & n.d. & n.d. & n.d. & n.d. & n.d. & n.d. & n.d. & n.d. & n.d. & n.d. & n.d. & n.d. \\
\hline 0.72 & 2.05 & 0.51 & 1521 & 125 & 2.75 & 10.4 & 7.4 & 2.8 & 72 & 10.3 & 7.0 & 2.5 & 261 \\
\hline
\end{tabular}


Table 5. Average sediment composition of Peru margin sediments and comparison with data from other upwelling sediments.

\begin{tabular}{|c|c|c|c|c|c|c|c|c|c|c|c|c|}
\hline & Opal & $\mathrm{CaCO}_{3}$ & TOC & TRS & $\mathrm{La}$ & $\mathrm{Ce}$ & $\mathrm{Sm}$ & $\mathrm{Eu}$ & $\mathrm{Yb}$ & Lu & $\mathrm{Ba}$ & $\mathrm{Cr}$ \\
\hline Number of Cases & 91 & 177 & 170 & 156 & 179 & 179 & 179 & 179 & 178 & 179 & 179 & 179 \\
\hline Minimum & 1.1 & 0.00 & 0.03 & 0.11 & 7 & 10 & 1.59 & 0.2 & 0.1 & 0.02 & 11 & 42 \\
\hline Maximum & 17.6 & 49.87 & 17.94 & 2.63 & 37 & 60 & 7.70 & 1.3 & 3.8 & 0.74 & 3252 & 196 \\
\hline Mean & 6.3 & 5.37 & 5.48 & 0.99 & 21 & 36 & 4.35 & 0.8 & 2.0 & 0.42 & 841 & 98 \\
\hline \multirow[t]{2}{*}{ Standard Dev. } & 3.8 & 7.04 & 3.19 & 0.44 & 6 & 11 & 1.12 & 0.2 & 0.6 & 1.22 & 567 & 29 \\
\hline & $\mathrm{Fe}$ & Cs & Co & Hf & $\mathrm{Rb}$ & $\mathrm{Sc}$ & $\mathrm{Th}$ & $\mathrm{Sb}$ & $\mathrm{Br}$ & & & \\
\hline Number of Cases & 179 & 179 & 179 & 179 & 179 & 179 & 179 & 179 & 179 & & & \\
\hline Minimum & 0.72 & 1.6 & 1.6 & 0.66 & 18.8 & 2.9 & 1.6 & 0.6 & 12 & & & \\
\hline Maximum & 5.77 & 14.5 & 11.75 & 9.28 & 118.4 & 16.9 & 10.5 & 4.2 & 390 & & & \\
\hline Mean & 2.77 & 8.1 & 6.77 & 3.13 & 72.5 & 10.7 & 6.5 & 1.9 & 143 & & & \\
\hline Standard Dev. & 0.89 & 3.2 & 2.26 & 1.15 & 22.1 & 3.1 & 2.1 & 0.6 & 88 & & & \\
\hline Component & $\mathrm{n}$ & TOC & $\mathrm{La}$ & $\mathrm{Ce}$ & $\mathrm{Ba}$ & $\mathrm{Cr}$ & $\mathrm{Fe}$ & Co & $\mathrm{Rb}$ & $\mathrm{Sc}$ & Th & $\mathrm{Br}$ \\
\hline${ }^{1}$ Peru upwelling & 179 & 5.5 & 21 & 36 & 840 & 98 & 2.8 & 6.7 & 72.5 & 10.7 & 6.5 & 143 \\
\hline${ }^{2}$ Gulf of California & 50 & 4.4 & n.g. & n.g. & 566 & 44 & 7 & n.g. & n.g. & n.g. & n.g. & n.g. \\
\hline${ }^{3}$ Benguela upwelling & 44 & 6.1 & 14 & 30 & 2927 & 101 & 3.2 & 11 & 63 & 22 & 6 & 104 \\
\hline${ }^{4}$ Average clay & n.g. & 0.3 & 115 & 345 & 2300 & 90 & 6.5 & 74 & 110 & 19 & 7 & 70 \\
\hline${ }^{5}$ Average carbonates & n.g. & n.g. & 10 & 35 & 190 & 11 & 0.9 & 1 & 10 & 2 & 1 & 70 \\
\hline
\end{tabular}

1 This study.

2 Brumsack, 1986.

3 Emeis, 1985.

4 Turekian and Wedepohl, 1961.

5 Turekian and Wedepohl, 1961. 